DOI: $10.21767 / 2171-6625.100072$

\title{
Pulmonary Arterial Hypertension in a Patient with Multiple Sclerosis Treated with Interferon Beta: A Case Report
}

\author{
Alena Novotna ${ }^{1}$, Edvard Ehler ${ }^{1,2}$, Petra Mandysova ${ }^{1,2}$ and Pavel Jansa ${ }^{3}$ \\ ${ }^{1}$ Neurology Clinic, Pardubice Hospital, Hospitals of the Pardubice Region, Pardubice, Czech Republic \\ ${ }^{2}$ Faculty of Health Studies, University of Pardubice, Pardubice, Czech Republic \\ ${ }^{3}$ Pulmonary Hypertension Centre, Second Internal Clinic - Clinic of Cardiology and Angiology, General University Hospital and the First Faculty of \\ Medicine of Charles University, Prague, Czech Republic
}

Corresponding author: Edvard Ehler, Associate Professor, MD, CSc, Neurology Clinic, Pardubice Hospital, Hospitals of the Pardubice Region, Kyjevská 44, Pardubice, 532 03, Czech Republic, Tel: +420 466014701; Fax: +420 466014702; E-mail: edvard.ehler@nemocnice-pardubice.cz

Received: Feb 20, 2016; Accepted: Feb 27, 2016; Published: Feb 29, 2016

\section{Abstract}

Pulmonary arterial hypertension (PAH) is characterized by elevation of mean pulmonary artery pressure to values conceivably approaching those seen in the systemic vascular bed. This occurrence is associated with a poor prognosis. PAH may result from a variety of causes, some of which are related to pharmacotherapy.

Herein, we present a case of a 39-year-old female patient with relapsing-remitting multiple sclerosis (MS), who was receiving immunomodulatory therapy and subsequently developed severe PAH. We presume a causal relationship between the condition and the patient's interferon beta therapy, which had spanned 10 years. The interferon beta therapy was stopped because its continued administration could have led to more rapid progression of severe pulmonary hypertension.

Due to the severity of pulmonary arterial hypertension, PAH-specific pharmacotherapy was indicated, using sildenafil. Since 1998, only several cases of pulmonary arterial hypertension in patients treated with interferon beta have been documented in the scientific literature. In some of these instances, including in two case studies involving patients with multiple sclerosis, it was concluded that there was a possible association with this therapy.

Keywords: Multiple sclerosis; Pulmonary arterial hypertension; Interferon beta sildenafil

\section{Introduction}

Pulmonary arterial hypertension (PAH) develops on the basis of pulmonary arterial wall remodeling, mainly on the basis of smooth muscle cell hypertrophy of the medial layer. Subsequent course of the disease is characterized by an increase in pulmonary vascular resistance, which leads to an elevation of pressure in the pulmonary vascular bed [1]. Normally, mean pulmonary artery pressure $(\mathrm{mmHg})$ is $\leq 20$
$\mathrm{mmHg}$. PAH is characterized by elevation of mean pulmonary artery pressure to values conceivably approaching those seen in the systemic vascular bed. The prognosis of the disease is poor, and most untreated individuals die within three years due to right-sided heart failure [2]

The predominant clinical signs and symptoms include progressive shortness of breath, considerable fatigability, chest pain, edema of the lower limbs, and other signs and symptoms of right-sided heart failure. Nonetheless, there are no absolutely typical signs and symptoms of PAH. All clinical features are nonspecific, which is, unfortunately, a frequent cause of delayed detection of the disease [3]. Luckily, however, the incidence of this disease is low-approximately 10 patients per million people [4].

For a definitive diagnosis to be made, right-sided heart catheterization with measurement of mean pulmonary artery pressure is required. $\mathrm{PAH}$ is considered mild when the pressure values range from $25-35 \mathrm{mmHg}$, moderate when they range from $36-45 \mathrm{mmHg}$, and severe when they exceed $45 \mathrm{mmHg}$. Echocardiography shows and quantifies right ventricular hypertrophy, and spirometry is used to diagnose non-specific functional disorder.

Treatment is decided based on the progression of symptoms, as evaluated according to the New York Heart Association (NYHA) classification system (NYHA class I-IV). Specific treatment of PAH includes administration of calciumchannel blockers that act as vasodilators, endothelin receptor antagonists, phosphodiesterase 5 (PDE5) inhibitors, and soluble guanylate cyclase stimulants.

Despite substantial diagnostic and therapeutic advancements, multiple sclerosis (MS) remains a serious, incurable autoimmune disease that affects and incapacitates mainly young people in the 20-40 age group. First-choice immunomodulatory drugs include interferon beta-1a (Rebif 22 $\mu \mathrm{g}$ and $44 \mu \mathrm{g}$ and Avonex), interferon beta-1b (Betaferon), and glatiramer acetate (Copaxone)-so far, this has been the only antigen-specific therapy of MS. These first-choice drugs continue to be a fundamental pillar of treatment of patients with clinically isolated syndrome and the relapsing-remitting form of multiple sclerosis [1]. 


\section{Case Report}

A 39-year-old patient with the relapsing-remitting form of MS had been diagnosed with MS at the age of 29 based on the clinical picture, a magnetic resonance imaging (MRI) scan, and cerebrospinal fluid findings. She had never been significantly ill. In 2005 (at the age of 29), she was placed on immunomodulatory therapy using Rebif $22 \mu \mathrm{g}$, which was administered subcutaneously three times per week. At the same time, she was kept on a low-dose oral corticosteroid (Medrol $8 \mathrm{mg}$ every other day). At initiation of biological therapy, the patient's Expanded Disability Status Scale (EDSS) score was 2.5 .

Because of increased disease activity, the patient's treatment was changed to Rebif $44 \mu \mathrm{g}$ in December 2012, at which time her EDSS score was 3.0. Her neutralizing antibody (NAb) titers were negative on repeated occasions. Subjectively, she complained of considerable fatigue. Slight paraparesis of the lower limbs, more so on the left side, was the predominant objective finding. A follow-up MRI scan in $03 / 2012$ did not confirm disease progression. MRI supplemented by images of the thoracic part of the spine, which was performed in $06 / 2013$, showed global patchy gliotic foci with traction on the spinal cord at the T7-T9 and T10-T11 levels. Thus, this MR image revealed the presence of older plaques of demyelination in MS The patient subsequently developed depressive episodes; therefore, she initiated therapy with a selective serotonin reuptake inhibitor (SSRI) and psychiatric follow-up.

Since the end of 2013, the patient had been noticing an increasing number of episodes of dyspnea, and she was subsequently examined in an ambulatory internal medicine clinic serving her catchment area. In 06/2014, there was a significant increase of her transient dyspnea, and a lung ventilation/perfusion (V/Q) scan established the diagnosis of pulmonary embolism, with complete resolution confirmed by a follow-up V/Q scan in 10/2014. In view of suspected chronic thromboembolic pulmonary hypertension, the patient was referred to the Second Internal Clinic of Cardiology and Angiology at the First Faculty of Medicine of Charles University and the General University Hospital in Prague, in order to complete the diagnostic work-up of pulmonary hypertension. The patient's hospitalization occurred in October 2014 Uncomplicated right-sided heart catheterization confirmed the presence of severe fixed pre-capillary pulmonary hypertension with low cardiac output. Subsequently, CT pulmonary angiography was performed, which was not indicative of chronic thromboembolic pulmonary hypertension (CTEPH). The etiological conclusion was that this was pulmonary arterial hypertension, and its development in association with interferon-beta treatment could not be excluded. Therefore, the recommendation was to discontinue its administration.

Based on the NYHA functional class III, specific pharmacotherapy was indicated, and already during her hospitalization, the patient was started on sildenafil $20 \mathrm{mg}$, at the dose of one-half $(1 / 2)$ tablet three times daily. Interferon beta treatment was discontinued. Overall duration of interferon beta administration had been 10 years. Since the cessation of interferon beta treatment, the patient had experienced, in January 2015, an exacerbation of her MS with predominant paraparesis of the lower limbs. Prior to the exacerbation, the patient had developed a rather severe bout of respiratory infection with a temporary worsening of dyspnea. She was treated with intravenous pulse Solu-Medrol at a dose of $4 \mathrm{gm}$. After the treatment, the patient's lower limb mobility and gait subsequently improved. She has been able to walk independently to a maximum distance of $300 \mathrm{~m}$; a longer distance has led to an increase of dyspnea and fatigue.

Currently, the patient's clinical findings show remission of the relapsing-remitting form of $\mathrm{MS}$, with a predominant residual paraparesis of the lower limbs and other multifocal symptoms from the central nervous system. The patient's last follow-up MRI scan of the brain and the thoracic part of the spine was performed in 04/2014, with no noticeable progression. The patient's current pharmacotherapy consists of intravenous immunoglobulins once per month and antidepressant therapy. She has been kept on long-term anticoagulant treatment with warfarin, at a dose of $5 \mathrm{mg}$ daily. Since the bout of respiratory infection that was treated in $01 / 2015$, the patient's daily dose of sildenafil has been increased to $60 \mathrm{mg}$.

\section{Discussion}

$\mathrm{PAH}$ usually affects younger women; frequently, the condition develops without any known cause. In general, it affects small arteries (plexiform lesions are typical), which is most likely caused by primary endothelial damage seen in the pulmonary vascular bed. The prognosis of patients with the disease is poor; most untreated patients die within three years due to right-sided heart failure. The main clinical signs and symptoms of $\mathrm{PAH}$ include progressive dyspnea with peripheral cyanosis, chest pains, and fatigability [4]. As for our patient, progressive dyspnea and fatigability had been among her principal initial signs and symptoms of PAH before a diagnosis was made. In predisposed patients, a causal relationship between the development of PAH and interferon is presumed, and hypothetically, its continued administration can lead to a swifter progression of pulmonary hypertension [5].

Right-sided heart catheterization produces a definitive diagnosis, with measurement of mean pulmonary artery pressure ( $25 \mathrm{mmHg}$ and above) and pulmonary artery wedge pressure (up to $15 \mathrm{mmHg}$ ), which was also the case with our patient [2]. Before the performance of the right-sided heart catheterization, heart disease, lung disease, and chronic thromboembolic pulmonary hypertension (CTEPH) were all considered to be possible causes of the patient's pulmonary hypertension; however, they were excluded in the process of differential diagnosis.

Based on the findings from the right-sided heart catheterization, which confirmed the presence of severe $\mathrm{PAH}$, the patient was immediately started on sildenafil at a dose of $30 \mathrm{mg}$ daily, in line with the current professional literature $[1,5,6]$. After three months of treatment, the dosage was 
increased to $60 \mathrm{mg}$ daily, following a bout of respiratory infection with accentuated dyspnea, which had been accompanied by an exacerbation of the patient's MS that had been treated with Solu-Medrol using pulse dosing.

As for MS and PAH -the scientific literature has described two cases of PAH development in patients with MS treated with interferon beta $1 \mathrm{a}$, administered subcutaneously three times per week. A 59-year-old patient developed PAH after a year-long treatment with interferon beta, which was subsequently discontinued, and the patient was started on sildenafil [5]. A very similar situation has been described in a case report of a 23-year-old woman treated with interferon beta, who developed PAH after one year of treatment as well; her interferon treatment was discontinued, and she was immediately placed on sildenafil. The patient's further prognosis was favorable $[1,2]$.

Unlike the two mentioned cases, our patient had been treated with interferon beta 1 a for a period of 10 years. She was diagnosed with PAH in October 2014. Due to accentuated dyspnea after a bout of respiratory infection in 01/2015, the patient's dose of sildenafil was increased to $60 \mathrm{mg}$, as already mentioned above. Unfortunately, the prognosis is very unfavorable.

\section{Conclusion}

Pulmonary arterial hypertension develops without a known cause; it manifests as progressive right-sided failure with predominant dyspnea, and the prognosis of the disease is poor. Secondary pulmonary hypertension develops as a result of a variety of other diseases. In the case of our patient, rightsided heart catheterization confirmed the presence of severe fixed pre-capillary pulmonary hypertension with low cardiac output. Despite an initial suspicion of a chronic thromboembolic cause, this diagnosis was not confirmed. Our patient probably developed pulmonary arterial hypertension in association with her long-term treatment of multiple sclerosis with interferon beta. Even with efficacious treatment of pulmonary arterial hypertension, the prognosis of the patient's disease is very unfavorable.

\section{Conflict of Interest}

I declare, that we have no conflict of interest.

\section{Acknowledgment and Financial Support}

We declare that there is no financial interest.

\section{Ethics}

The work described in this case report has been conducted in accordance with the World Medical Association's Declaration of Helsinki - Ethical principles for medical research involving human subjects, and with the journal's publishing ethics, as specified on the Elsevier Publishing Campus.

\section{References}

1. Rot U, Ledinek AH (2013) Interferons beta have vasoconstrictive and procoagulant effects: a woman who developer livedo reticularis and Raynaud phenomenon in association with interferon beta treatment for multiple sclerosis. Clinical Neurology and Neurosurgery; 115: S79-S81.

2. Ledinek AH, Jazbec SS, Drinovec I, Rot U (2009) Pulmonary arterial hypertension associated with interferon beta treatment for multiple sclerosis: a case report. Multiple Sclerosis 15: 885-886.

3. Ietra GG, Capron F, Stewart S, Leone O, Humbert M, et al. (2004) Pathologic assessment of vasculopathies in pulmonary hypertension. J Am Coll Cardiol 43: 25S-32S.

4. Kiss T, Kovacs K, Komocsi A, Tornyos A, Zalan P, et al. (2014) Novel mechanisms of sildenafil in pulmonary hypertension involving cytokines/chemokines, MAP kinases and Akt. PLoS ONE 9(8): e104890.

5. Caravita S, Secchi BM, Wu SC, Pierini S, Paggi A (2011) Sildenafil therapy for interferon- $\beta$-1a-induced pulmonary arterial hypertension: a case report. Cardiology 120: 187-189.

6. Brenot F, Herve P, Petitpretz P, Parent F, Duroux P, Simonneau G (1993) Primary pulmonary hypertension and fenfluramine use. Br Heart J 70: 537-541. 